als mir zuerst die Berührung des Mondrandes mit dem Planeten auffiel. Das gänzliche Verschwinden des letzteren notirte ich $2 u 8^{\mathrm{h}} 3^{\mathrm{m}} 44^{\mathrm{s}} \cdot 75$, Frau Manora zu $\left.8^{\mathrm{h}} 4^{\mathrm{m}} 44^{\mathrm{s}} \cdot 5^{*}\right)$. Das Wiederauftauchen des Planeten notirte ich $2 \mathrm{u} 8^{\mathrm{h}} 41^{\mathrm{m}} \times 5^{\mathrm{s}} \cdot 5$, (es erfolgte zwischen zwei Mondbergen) und seine Loslösung vom Mondrand $2 \mathrm{u} 8^{\mathrm{h}} 4^{\mathrm{m}} 3^{\mathrm{s}}$. Frau Manora notirte $8^{\mathrm{h}} 41^{\mathrm{m}} 16^{\mathrm{s}}$ bezw. $8^{\mathrm{h}} 42^{\mathrm{m}} 3^{5} \cdot 5$.

Zwischen den beiden ersten und den beiden letzten
Contacten beobachteten wir die Bedeckung von 132 Tauri und zwar insofern unter günstigeren Umständen, als sich die Dunstwolke mittlerweile verzogen hatte und die Nachtseite des Mondes in beiden Fernrohren sehr scharf zu sehen war. Ich notirte das plötzliche Verschwinden des Sterns (ohne merkliche Lichtabnahme) um $8^{\mathrm{h}} \mathbf{3 2}^{\mathrm{m}} 3^{\mathrm{s}} \cdot 5$, Frau Manora um $8^{\mathrm{h}} 32^{\mathrm{m}} 3^{5} \cdot 25$. Das Wiederauftauchen konnte wegen plötzlich eintretender Bewölkung nicht beobachtet werden.

Manora-Sternwarte, Lussinpiccolo, Istrien, 1898 Mai 23.

Leo Brenner.

*) Offenbar in Folge Versehens beim Ablesen der Minuten, statt $3^{\mathrm{m}}$.

\title{
Observations d'éclipses des Satellites de Jupiter et d'occultation de Venus.
}

Dans le tableau suivant des moments observés de disparition (D) ou de réapparition (R) des satellites de Jupiter, le nombre ajouté à l'initiale de l'observateur (Geelmuyden ou Schroeter) indique l'ouverture, en centimètres, de l'instrument employé.

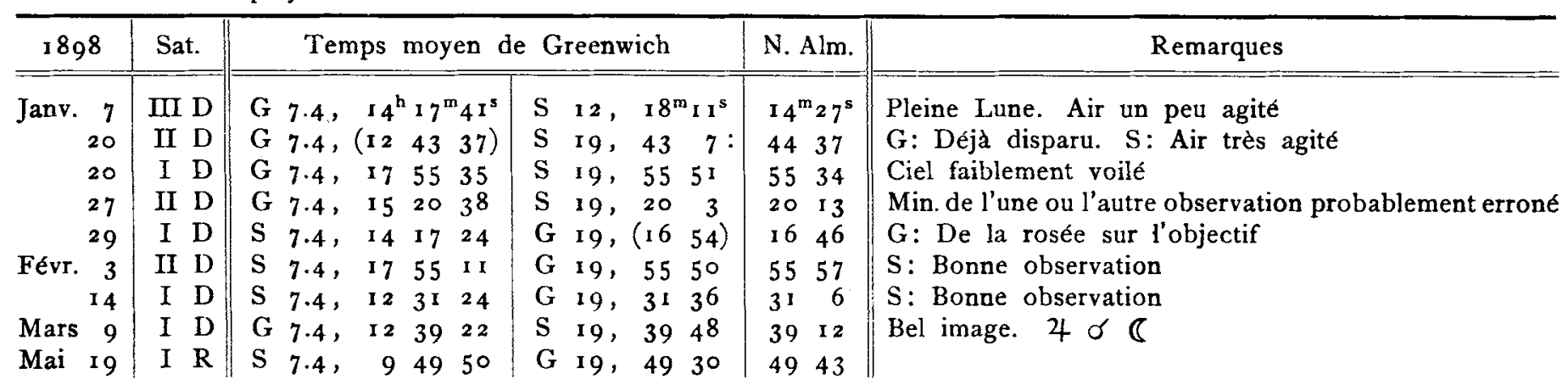

L'occultation de Venus 1898 Mai 22 a été observé comme il suit:

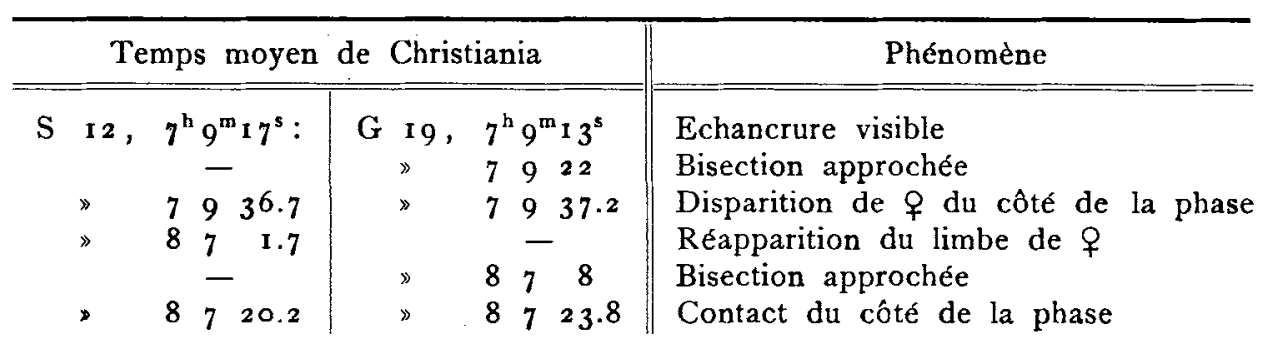

L'immersion fut observée à travers des nuages flottants; le bord illuminé de la Lune était invisible dans les deux instruments. A l'émersion l'éclat de Venus était fort supérieur à celui de la Lune.

Les coordonnées des deux instrument sont:

Latitude

Longitude (Comp. de Bakhuyzen)

Hauteur au dessus de la mer

Christiania le 27 mai 1898 .

$$
\begin{aligned}
& \text { Equatoréal de Repsold } \\
& \text { I } 2 \mathrm{~cm} \text {, gross. } 5^{\circ} \\
& 59^{\circ} 54^{\prime} 44^{\prime \prime} \\
& 4^{\mathrm{m}} 53^{\mathrm{s}} \cdot 5^{6} \\
& 35 \mathrm{~m}
\end{aligned}
$$

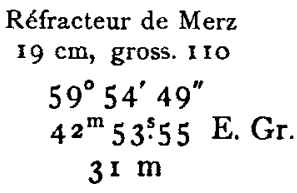

Réfracteur de Merz $19 \mathrm{~cm}$, gross. 110

$59^{\circ} 54^{\prime} 49^{\prime \prime}$ $42^{\mathrm{m}} 53^{\mathrm{s}} \cdot 55$ E. Gr.

\begin{tabular}{|c|c|c|c|c|c|c|c|c|c|c|}
\hline 1898 & M.Z.Wien & $\Delta \alpha$ & $\Delta \delta$ & Vgl. & $\alpha$ app. & $\log p . \Delta$ & $\delta$ app. & $\log p .4$ & Red. ad 1. app. & $*$ \\
\hline 21 & $16^{\mathrm{h}} 31^{\mathrm{m}} 18^{\mathrm{s}}$ & $+1^{m} 1^{5}: 00$ & $+317: 6$ & 6 & $2 \mathrm{I}^{\mathrm{h}} 24^{\mathrm{m}} 28.76$ & $9.600_{n}$ & $+18^{\circ} 23^{\prime} 37^{\prime \prime} 9$ & 0.757 & $+0.42-6.0$ & \\
\hline 27 & $16 \quad 8 \quad 34$ & -o 33.03 & $-0 \quad 33.9$ & 6 & $2147 \quad 26.14$ & $9.629 n$ & +243353.4 & $0.74^{1}$ & $+0.35-5.6$ & \\
\hline 28 & $15 \quad 57 \quad 49$ & -227.80 & +257.2 & 6 & $\begin{array}{lll}21 & 51 & 24.84\end{array}$ & $9.6 \sigma_{n}$ & +253421.2 & 5 & $.33-5 \cdot 4$ & \\
\hline 31 & $16 \quad 839$ & +251.41 & +6 & 6 & $\begin{array}{lll}22 & 3 & 49.00\end{array}$ & $9.644 n$ & +28348.4 & 0.720 & $+0.31-5.5$ & \\
\hline
\end{tabular}
$3 \mathrm{I} \mathrm{m}$

H. Geelmuyden.

\section{Beobachtungen des Cometen 1898 I}

am $6 z$. Fraunhofer'schen Refractor der k. k. Sternwarte in Wien von Dr. F. Holetschek. 
Mittlere Oerter der Vergleichsterne für 1898.0.

\begin{tabular}{|c|c|c|c|c|c|c|c|}
\hline$*$ & $\alpha$ & $\delta$ & Autorität & * & $\alpha$ & $\delta$ & Autorität \\
\hline $\begin{array}{l}1 \\
2\end{array}$ & $\begin{array}{l}21^{h} 23^{m} 27^{s}: 34 \\
2147 \\
58.82\end{array}$ & $\begin{array}{l}+18^{\circ} 20^{\prime} 26^{\prime \prime} 3 \\
+243432.9\end{array}$ & $\begin{array}{l}\text { AG. Berlin A. } 8759 \\
\text { AG. Berlin B. } 8438\end{array}$ & $\begin{array}{l}3 \\
4\end{array}$ & $\begin{array}{l}21^{\mathrm{h}} 53^{\mathrm{m}} 5^{2^{\mathrm{s}}} 3^{\mathrm{I}} \\
22 \quad \circ 57.28\end{array}$ & $\begin{array}{r}+25^{\circ} 3 I^{\prime} 29^{\prime \prime} 4 \\
+28285.5\end{array}$ & $\begin{array}{l}\text { AG. Cambr. I } 3058 \\
\text { AG. Cambr. } 13175\end{array}$ \\
\hline
\end{tabular}

Beobachtungen über den Grad der Wahrnehmbarkeit (Gesammthelligkeitseindruck) und über die Schweiflänge des Cometen.

$M$ ist die beobachtete, $M_{1}$ die durch Subtraction von $5 \log r \Delta$ auf $r=1, \Delta=$ reducirte Grössenclasse. Die Vergleichungen von März 28 und 31 sind mit einem Opernglase, alle übrigen bis zum Juni mit dem kleinen $11 / 2$. Sucher gemacht.

\begin{tabular}{c|r|r|r|r|r}
\hline 1 898 & $\log r$ & $\log \Delta$ & $5 \log r \Delta$ & $M$ & $M_{1}$ \\
\hline März 2 1, 23 & 0.04 & 0.20 & +1.2 & 6.7 & 5.5 \\
26 & 0.05 & 0.20 & 1.2 & 6.7 & 5.5 \\
27 & 0.05 & 0.20 & 1.2 & 6.1 & 4.9 \\
28 & 0.05 & 0.20 & 1.2 & 5.7 & 4.5 \\
3 1 & 0.05 & 0.20 & 1.3 & 5.6 & 4.3 \\
April 5 & 0.06 & 0.21 & 1.3 & 6.0 & 4.7 \\
8 & 0.06 & 0.21 & 1.4 & 6.4 & 5.0 \\
I 1 & 0.07 & 0.22 & 1.4 & 6.4 & 5.0 \\
1 4 & 0.08 & 0.22 & 1.5 & 6.3 & 4.8 \\
I6 & 0.08 & 0.23 & 1.5 & 6.4 & 4.9 \\
26, 27 & 0.11 & 0.26 & +1.8 & 6.8 & 5.0
\end{tabular}

In den folgenden Angaben über die scheinbare Schweiflänge bedeutet die erste der Columnen $C$ die Länge, bis zu welcher der Schweif ganz deutlich zu sehen war, die zweite die äusserste Länge, bis zu welcher er an Tagen mit besonders klarer Luft noch einigermaassen sicher verfolgt -

\begin{tabular}{rll} 
I 898 & \multicolumn{2}{c}{$C$} \\
März 2 I & $10^{\circ}$ & $60^{\prime}$ \\
27 & 20 & 60 \\
29 & 30 & - \\
April I 4 & 20 & -
\end{tabular}

Diesen Beobachtungen zufolge war die Helligkeit des Cometen in der zweiten und dritten Woche nach dem Perihel ( $T=$ März I 7 ) am grössten, und auch der Schweif scheint in dieser Zeit (wobei jedoch Anfang April des Mondscheins wegen vom Schweif nicht viel gesehen werden konnte) am

Wien, 1898 Juni 27.

\begin{tabular}{|c|c|c|c|c|c|}
\hline 1898 & $\log r$ & $\log A$ & $5 \log r A$ & $M$ & $M_{1}$ \\
\hline April 3 & 0.12 & 0.27 & +1.9 & $7 \cdot 3$ & 5.4 \\
\hline Mai & 0.12 & 0.27 & 1.9 & 7.4 & $5 \cdot 5$ \\
\hline $\mathbf{I}$ & 0.16 & $0.3^{1}$ & $2 \cdot 3$ & 7.8 & $5 \cdot 5$ \\
\hline $\mathbf{I}$ & 0.16 & $0.3^{1}$ & 2.4 & 8.0 & 5.6 \\
\hline I & 0.16 & $0.3^{2}$ & 2.4 & 7.9 & $5 \cdot 5$ \\
\hline I & 0.17 & $0.3^{2}$ & 2.5 & 7.9 & 5.4 \\
\hline 2 & 0.18 & 0.33 & 2.6 & 8.4 & 5.8 \\
\hline 2 & 0.18 & 0.34 & 2.6 & 8.6 & 6.0 \\
\hline 2 & 0.18 & 0.34 & 2.6 & 8.5 & $5 \cdot 9$ \\
\hline 2 & 0.19 & 0.34 & 2.7 & 8.6 & 5.9 \\
\hline Juni & 0.23 & $0.3^{8}$ & +3.1 & 9.5 & 6.4 \\
\hline
\end{tabular}

werden konnte. Auch sei bemerkt, dass ich bezuglich der Schweiflänge zwischen den Beobachtungen mit dem $6 \mathrm{z}$. Refractor und dem $I^{1 / 2} z$. Sucher an den meisten Tagen keinen wesentlichen Unterschied gefunden habe.

\begin{tabular}{rrrr}
\multicolumn{2}{c}{1898} & \multicolumn{2}{c}{$C$} \\
April 16 & $25^{\circ}$ & - \\
& 27 & 10 & $20^{\circ}$ \\
Mai & 30 & 5 & - \\
& 1 & 5 & -
\end{tabular}

längsten und hellsten gewesen zu sein. Mit dem entschiedenen Zurückgehen der Schweifbildung Ende April und Anfang Mai hat auch die reducirte Grösse $M_{1}$ wesentlich abgenommen und in der ersten Hälfte des Mai wieder den Werth erreicht, den sie am Anfang der Beobachtungen gezeigt hat.

\section{Aggiunta alla Rettifica in Astr. Nachr. 3512.}

In seguito ad odierna amichevole comunicazione del Prof. Kreutz circa la stella $W_{1} 5^{\mathrm{h}} \cdot \mathbf{r}^{6}$, la cui recente posizione, aequ. 1900.0:

$$
a=5^{\mathrm{b}} 10^{\mathrm{m}} 3^{2} \cdot 3 \circ \quad \delta=-2^{\circ} 44^{\prime} 57^{\prime \prime} 4
$$

il Prof. Becker ebbe la bontà di concludere da due osservazioni meridiane di Strassburgo del 1889.07 , ho potuto sostituire alla posizione della stella r ro delle mie osservazioni, A. N. vol. I 46 pag. 62 , quest' altra :

$$
\alpha(1897)=5^{\mathrm{h}} 10^{\mathrm{m}} 23^{\mathrm{s} .27} \quad \delta(1897)=-2^{\circ} 45^{\prime} 10^{\prime \prime} 3
$$

Arcetri 1898 Sett. I e ricavare gli $\mathrm{O}-\mathrm{C}$ di (6) Ebe seguenti :

$\begin{array}{rcc}1897 & \Delta \alpha & \Delta 8 \\ \text { Nov. } 22 & +1.80 & +7.9 \\ 23 & +1.78 & +9.1\end{array}$

i quali non discordano più, nè da quelli del Dr. Antoniazzi (A. N. vol. 146 pag. 330) nè da quelli del Dr. Viaro (Pubbl. di Arcetri Fasc. 8), di tanto quanto puo essere imputato ad equazione personale ed instrumentale. 